\title{
Descontinuación del esteroide inhalado en pacientes con enfermedad pulmonar obstructiva crónica moderada a severa no aumenta el riesgo de exacerbaciones
}

\section{Discontinuation of inhaled steroid in patients with moderate-to-severe COPD does not increase the risk of exacerbation}

Carlos Andrés Celis Preciado, MD.

Internista, Neumólogo, Hospital Universitario San Ignacio, Pontificia Universidad Javeriana, Bogotá, Colombia.

Correspondencia: Carlos Celis Preciado, correo electrónico: cacelis.neumo@gmail.com Recibido: 16/08/14. Aceptado: 10/09/14.

\section{Referencia}

Magnussen H, Disse B, Rodriguez-Roisin R, Kirsten A, Watz $\mathrm{H}$, Tetzlaff $\mathrm{K}$, et al; WISDOM Investigators. Withdrawal of inhaled glucocorticoids and exacerbations of COPD. N Engl J Med. 2014;371(14):1285-94.

\section{Pregunta}

En pacientes con enfermedad pulmonar obstructiva crónica (EPOC) que reciben triple terapia con anticolinérgico de larga acción (ACLA), beta 2 agonista de larga acción (BALA) y esteroide inhalado, ¿es la descontinuación del esteroide inhalado, en comparación con su continuación, no inferior en cuanto a prevención de exacerbaciones?

\section{Diseño}

Estudio de asignación aleatoria, de no inferioridad. WISDOM (del inglés Withdrawal of Inhaled Steroids during Optimized Bronchodilator Management).

\section{Asignación y cegamiento}

Enmascarada, con cegamiento de pacientes y médicos.

\section{Sitios y seguimiento}

Se llevó a cabo en 200 centros clínicos en 23 países, a lo largo de 52 semanas.

\section{Pacientes}

Se enrolaron 2.488 pacientes con EPOC severa $(61 \%)$ y muy severa $(38 \%)$ y al menos una exacerbación en el año previo (1.537 tuvieron una exacerbación y 903 dos o más). Edad promedio 64 años, 83\% hombres, $65 \%$ exfumadores, con $\mathrm{VEF}_{1}$ postbroncodilatador promedio de 0,97 litros (34\% del predicho), disnea mMRC 1,8 y puntaje SGRQ (del inglés St George's Respiratory Questionnaire) de 46. El 70\% recibía esteroide inhalado, $65 \%$ BALA, $47 \%$ ACLA y $38 \%$ los tres medicamentos. En cuanto a comorbilidades, 28,2\% tenían trastornos cardíacos, $45,8 \%$ vasculares y $40 \%$ hipertensión arterial. 
Se excluyeron pacientes con exacerbaciones en las seis semanas previas, empleo de oxígeno diurno, rehabilitación pulmonar en las últimas cuatro semanas, asma, bronquiectasias, antecedente de resección pulmonar, neoplasia en tratamiento $\mathrm{o}$ antecedente de infarto del miocardio o arritmia en los tres meses previos u hospitalización por falla cardiaca en el año anterior.

Los participantes completaron un periodo inicial de seis semanas con tiotropio $18 \mathrm{mcg} /$ día, salmeterol $50 \mathrm{mcg}$ cada 12 horas y fluticasona 500 mcg cada 12 horas.

\section{Intervención}

1.243 participantes fueron asignados a continuación de la triple terapia por 52 semanas y 1.244 a descontinuación progresiva del esteroide inhalado cada seis semanas, de una dosis total de 1.000 mcg de fluticasona a $500 \mathrm{mcg}$, después a $200 \mathrm{mcg}$ y finalmente a $0 \mathrm{mcg}$, y a permanecer sin el esteroide inhalado por el resto de las cuarenta semanas.

\section{Desenlaces}

- Primario: Tiempo a la primera exacerbación moderada o severa a los doce meses. Límite superior del margen de no inferioridad 1,20 (poder $90 \%$, alfa 1 cola: 0,025 ).
- Secundarios: disnea (mMRC), función pulmonar (cambio en el $\mathrm{VEF}_{1}$ valle), calidad de vida (SGRQ) y tiempo a la primera exacerbación severa.

\section{Seguimiento}

$81 \%$ de los participantes completó el estudio y se realizó un análisis por intención a tratar.

\section{Resultados}

La descontinuación del esteroide inhalado no fue inferior con relación al riesgo de exacerbaciones moderadas a severas, exacerbaciones severas, cambio en disnea o calidad de vida. El grupo que suspendió el esteroide inhalado mostró una reducción de $43 \mathrm{ml}$ en el $\mathrm{VEF}_{1}$ valle $(\mathrm{p}=0,001)$ (tabla 1). No se observaron diferencias entre los grupos con relación a eventos adversos serios o fatales, cardiovasculares o neumonía.

\section{Conclusiones}

En pacientes con EPOC moderada a severa y antecedente de una exacerbación en el último año que reciben terapia triple (tiotropio+salmeterol+fluticasona), la descontinuación del esteroide inhalado no fue inferior a continuarlo para prevenir el riesgo

Tabla 1. Desenlaces primarios y secundarios del estudio WISDOM.

\begin{tabular}{|c|c|c|c|}
\hline Desenlace & Suspensión EI & Continuación EI & Comentario \\
\hline $\begin{array}{l}\text { Tiempo a la primera exacerbación } \\
\text { moderada o severa }\end{array}$ & -- & -- & HR $1,20($ IC95\%, $0,98-$ \\
\hline $\begin{array}{l}\text { Riesgo de exacerbación moderada } \\
\text { o severa }\end{array}$ & $47 \%$ & $44 \%$ & $\begin{array}{r}\text { HR } 1,06 \text { (IC95\% 0,94- } \\
1,19), p=0,35\end{array}$ \\
\hline $\begin{array}{l}\text { Tasa ajustada de exacerbación } \\
\text { moderada o severa }\end{array}$ & 0,95 (IC95\% 0,87-1,04) & 0,91 (IC95\%0,83-0,99) & \\
\hline Disnea (mMRC) & $+0,035$ & $-0,028$ & $p=0,06$ \\
\hline Calidad de vida SGRQ) & $+1,15$ & $+0,07$ & $p=0,047$ \\
\hline $\mathrm{VEF}_{1}$ & & & $+43 \mathrm{ml}(p<0,001)$ \\
\hline Eventos adversos serios & $24,2 \%$ & $23,5 \%$ & NS \\
\hline Neumonía & $5,5 \%$ & $5,8 \%$ & NS \\
\hline
\end{tabular}


de exacerbaciones moderadas a severas; no se observó diferencia con relación a disnea, calidad de vida, exacerbaciones severas, neumonía o eventos adversos. Los datos si mostraron una disminución en el $\mathrm{VEF}_{1}$ a lo largo del seguimiento.

\section{Comentario}

Según la última actualización de la iniciativa GOLD (del inglés Global Strategy for the Diagnosis, Management and Prevention of COPD), los esteroides inhalados están indicados en pacientes con EPOC con limitación del flujo aéreo grave o muy grave $\left(\mathrm{VEF}_{1}<50 \%\right.$ del predicho) y/o exacerbaciones frecuentes que no se controlan adecuadamente con broncodilatadores de acción prolongada (Evidencia A), al reducir el riesgo de nuevas exacerbaciones; así mismo, se han recomendado para el tratamiento del síndrome de solapamiento asma/EPOC (ACOS) y se ha señalado que no deben usarse como monoterapia en los pacientes con EPOC (1). No obstante, en los últimos años se ha producido una reevaluación de su papel en el tratamiento de la enfermedad (2).

El estudio WISDOM mostró que en pacientes con EPOC con síntomas leves a moderados (puntuación de disnea $\mathrm{mMRC}<2$ ), pero con función pulmonar baja $\left(\mathrm{VEF}_{1}<50 \%\right)$ y menos de dos exacerbaciones en el último año, se podría considerar una descontinuación progresiva del esteroide inhalado a partir de triple terapia, al no aumentar el riesgo de exacerbaciones o de deterioro de síntomas, calidad de vida o eventos adversos. No obstante, en la semana 18, tras la retirada del esteroide, el descenso promedio del $\mathrm{VEF}_{1}$ valle fue 38 $\mathrm{ml}$ mayor $(\mathrm{p}<0,001)$ y $43 \mathrm{ml}(\mathrm{p}=0,001)$ al final de las 52 semanas de seguimiento (3-8).

Entre las limitaciones del estudio se han señalado (3-8):

- Seguimiento de menos de un año, probablemente no lo suficientemente largo para evaluar exacerbaciones dado que dichos eventos ocurren con una frecuencia promedio de 1,3 a 2 por año, lo cual es importante si se tiene en cuenta la tendencia observada de mayor riesgo de exacerbaciones severas en el grupo de descontinuación $(\mathrm{p}=0,08)$.

- El empleo del tiempo hasta la primera exacerbación, en lugar de la frecuencia de las exacerbaciones, como desenlace primario, teniendo en cuenta que el tiempo libre de esteroides en el grupo de retirada fue sólo de nueve meses.

- No análisis separado de los pacientes según la gravedad de la EPOC para evaluar respuesta diferencial.

- No estratificación de los pacientes según recuento de eosinófilos en sangre o en esputo, elevados hasta en $30 \%$ de los pacientes con EPOC y que ha demostrado predecir la respuesta al esteroide inhalado.

- El racional para el protocolo de descontinuación (pasando de 1.000 a 500 a $200 \mathrm{mcg}$ al día cada seis semanas) no está claro y este podría ser no práctico para su uso en la experiencia diaria.

- El criterio de inclusión que dice que los pacientes deberían haber tenido una o más exacerbaciones de EPOC en el año anterior. Dado que el $70 \%$ de los sujetos finalmente incluidos en el estudio ya venían en tratamiento con esteroide inhalado, implicaría que los pacientes sin exacerbaciones durante el empleo del esteroide inhalado fueran excluidos del estudio, grupo que, en teoría, podría beneficiarse más de su retiro, incluyendo así a los que al parecer no estaban bien controlados con el esteroide inhalado.

- Si bien hubo una diferencia significativa en la tasa de disminución del $\mathrm{VEF}_{1}$ a favor de los pacientes que continuaron el esteroide inhalado, se ha considerado que esta no fue clínicamente relevante. Sin embargo, se ha manifestado desacuerdo con esta interpretación, ya que el $\mathrm{VEF}_{1}$ promedio de estos pacientes al ingresar fue de tan sólo $900 \mathrm{ml}$, lo cual indica que el tratamiento con esteroide inhalado podría contribuir a reducir la disminución excesiva de la función pulmonar en la EPOC. 
Con relación a este último aspecto, los mecanismos por medio de los cuales los esteroides inhalados podrían preservar la función pulmonar, aún no están claros, y se describen al menos tres: potenciación de la broncodilatación inducida por los beta agonistas al aumentar el número de receptores beta 2 en la pared bronquial, efecto antiexudativo al reducir el edema de pared e inducción de vasoconstricción pulmonar al disminuir la liberación de mediadores proinflamatorios (8).

Recientemente se revisó el concepto de descenso del tratamiento en la EPOC (9) y tres trabajos adicionales reportaron información acerca de la descontinuación de los esteroides inhalados (10-12).

Dos de ellos, INSTEAD y OPTIMO, uno aleatorio y otro una cohorte prospectiva de efectividad, respectivamente, señalan que en pacientes con EPOC moderada y menos de dos exacerbaciones en el último año, la suspensión del esteroide inhalado no se relaciona con deterioro de la función pulmonar, la disnea, la calidad de vida o aumento de las exacerbaciones a lo largo de 26 semanas de seguimiento (10-11).

Por su parte, el COSMIC, de diseño aleatorio y llevado a cabo en sujetos con EPOC moderada a severa e historia de dos exacerbaciones, reporta que la descontinuación del esteroide inhalado se asoció con deterioro del $\mathrm{VEF}_{1}$ y la disnea, así como con el aumento de exacerbaciones leves y síntomas nocturnos (12).

El estudio de Magnussen y cols. sin duda podría tener implicaciones prácticas. Un principio básico en la medicina es tratar a los pacientes con la mínima cantidad de medicamentos necesarios para alcanzar los objetivos clínicos. Estos datos cobran especial relevancia al tener presente la evidencia acumulada acerca de los efectos adversos asociados con el empleo de esteroides inhalados en el paciente con EPOC, tales como aumento del riesgo de neumonía (13), si bien no de la mortalidad asociada (14), incremento en el riesgo de tuberculosis (15), micobacterias no tuberculosas (16), influenza (17), diabetes (18), cataratas (19), osteoporosis y candidiasis orofaríngea (20).
Se esperan nuevos estudios clínicos que permitan precisar el grupo que obtendrá el mayor beneficio del uso crónico de esteroides inhalados en pacientes con EPOC (21).

\section{Conflictos de interés}

El autor declara no tener conflictos de interés.

\section{Bibliografía}

1. From the Global Strategy for the Diagnosis, Management and Prevention of COPD, Global Initiative for Chronic Obstructive Lung Disease (GOLD) 2015. Disponible en: http://www.goldcopd.org/ Fecha de acceso: 10/09/14.

2. Babu KS, Kastelik JA, Morjaria JB. Inhaled corticosteroids in chronic obstructive pulmonary disease: a pro-con perspective. Br J Clin Pharmacol. 2014;78(2):282-300.

3. Halpin DM, Quint JK. The WISDOM of inhaled corticosteroids in COPD. Thorax. 2014;69(12):1071-2.

4. Agustí A. Inhaled steroids in COPD: Reasons for a debate. Rev Port Pneumol (2006). 2015;21(4):175-7.

5. Laniado-Laborín R. Discontinuing inhaled steroids might not be safe in severe COPD cases. Evid Based Med. 2015;20(2):57.

6. Stanbrook MB. ACP journal club. For preventing exacerbations of COPD, withdrawal of inhaled glucocorticoids was noninferior to continuation. Ann Intern Med. 2015;162(6):JC5.

7. Balkissoon R. Journal club. J COPD F. 2015; 2(1): 85-90.

8. Rodriguez Roisin R, Arismendi E. Inhaled corticosteroids withdrawal in severe patients with chronic obstructive pulmonary disease: a wisdom decision? Arch Bronconeumol. 2015;51(2):57-8.

9. Cazzola M, Rogliani P, Matera MG. Escalation and de-escalation of therapy in COPD: myths, realities and perspectives. Drugs. 2015. [Epub ahead of print].

10. Rossi A, van der Molen T, del Olmo R, Papi A, Wehbe L, Quinn $\mathrm{M}$, et al. INSTEAD: a randomised switch trial of indacaterol versus salmeterol/fluticasone in moderate COPD. Eur Respir J. 2014;44(6):1548-56.

11. Rossi A, Guerriero M, Corrado A. Withdrawal of inhaled corticosteroids can be safe in COPD patients at low risk of exacerbation: a real-life study on the appropriateness of treatment in moderate COPD patients (OPTIMO). Respir Res. 2014;15:77.

12. Wouters EF, Postma DS, Fokkens B, Hop WC, Prins J, Kuipers AF, Pasma HR, Hensing CA, Creutzberg EC; COSMIC (COPD and Seretide: a Multi-Center Intervention and Characterization) Study Group. Withdrawal of fluticasone propionate from combined salmeterol/fluticasone treatment in patients with COPD causes immediate and sustained disease deterioration: a randomised controlled trial. (COPD and Seretide: a Multi-Center Intervention and Characterization) Study Group. Thorax. 2005;60(6):480-7.

13. Kew KM, Seniukovich A. Inhaled steroids and risk of pneumonia for chronic obstructive pulmonary disease. Cochrane Database Syst Rev. 2014;3:CD010115.

14. Festic E, Scanlon PD. Incident pneumonia and mortality in patients with chronic obstructive pulmonary disease. A double effect of inhaled corticosteroids? Am J Respir Crit Care Med. 2015;191(2):141-8. 
15. Ni S, Fu Z, Zhao J, Liu H. Inhaled corticosteroids (ICS) and risk of mycobacterium in patients with chronic respiratory diseases: a metaanalysis. J Thorac Dis. 2014;6(7):971-8.

16. Andréjak C, Nielsen R, Thomsen VØ, Duhaut P, Sørensen HT, Thomsen RW. Chronic respiratory disease, inhaled corticosteroids and risk of non-tuberculous mycobacteriosis. Thorax. 2013;68(3):256-62.

17. Dong YH, Chang CH, Lin Wu FL, Shen LJ, Calverley PM, Löfdahl $\mathrm{CG}$, et al. Use of inhaled corticosteroids in patients with COPD and the risk of TB and influenza: A systematic review and meta-analysis of randomized controlled trials. Chest. 2014;145(6):1286-97.

18. Gläser S, Krüger S, Merkel M, Bramlage P, Herth FJ. Chronic obstructive pulmonary disease and diabetes mellitus: a systematic review of the literature. Respiration. 2015;89(3):253-64.

19. Weatherall M, Clay J, James K, Perrin K, Shirtcliffe P, Beasley R. Dose-response relationship of inhaled corticosteroids and cataracts: a systematic review and meta-analysis. Respirology. 2009;14(7):983-90

20. Battaglia S, Cardillo I, Lavorini F, Spatafora M, Scichilone N. Safety considerations of inhaled corticosteroids in the elderly. Drugs Aging. 2014;31(11):787-96.

21. Agusti A, Fabbri LM. Inhaled steroids in COPD: when should they be used? Lancet Respir Med. 2014;2(11):869-71. 